\title{
Angioplasty was no better than medication for reducing blood pressure or slowing disease progression in renal artery stenosis
}

van Jaarsveld BC, Krijnen P, Pieterman H, et al, for the Dutch Renal Artery Stenosis Intervention Cooperative Study Group. The effect of balloon angioplasty on hypertension in atherosclerotic renal-artery stenosis. $N$ Engl J Med 2000 Apr 6;342:1007-14.

QUESTION: In patients with atherosclerotic renal artery stenosis who have normal or mildly impaired renal function, is balloon angioplasty as effective as antihypertensive drug treatment for reducing blood pressure?

\section{Design}

Randomised (allocation concealed*), blinded \{outcome assessors $\} \nmid, *$ controlled trial with 12 months follow up.

\section{Setting}

26 clinical centres in the Netherlands.

\section{Patients}

106 patients (mean age $60 \mathrm{y}, 62 \%$ men) with renal artery stenosis measured by renal arteriography (reduction in luminal diameter $\geqslant 50 \%$ ) and normal to mildly impaired renal function (serum creatinine level $\leqslant 2.3 \mathrm{mg} / \mathrm{dl}[200$ $\mu \mathrm{mol} / \mathrm{l}])$. Exclusion criteria were cancer, hypertension caused by a condition other than renovascular disease, unstable coronary artery disease, heart failure, or pregnancy. Follow up was $98 \%$.

\section{Intervention}

56 patients were allocated to angioplasty and 50 patients to drug treatment. Patients in the angioplasty group stopped taking medication before angioplasty, took aspirin starting the day before the procedure, and continued for the next 6 months. Antihypertensive drugs could be started during follow up. At 3 months, patients in the angioplasty group could have repeated angioplasty, stent deployment, or bypass surgery if their diastolic blood pressure (BP) was $\geqslant 95 \mathrm{~mm} \mathrm{Hg}$ or serum creatinine concentrations had increased by $\geqslant 0.2 \mathrm{mg} / \mathrm{dl}$ $(20 \mu \mathrm{mol} / \mathrm{l})$; also given 1 of these conditions, patients in the medication alone group received increased doses of the drugs they had been using before randomisation, and angioplasty could be done when high $\mathrm{BP}$ or signs of impairment of renal function were present.

\section{Main outcome measures}

The primary outcome was BP. Secondary outcomes were renal function and number and daily dose of antihypertensive drugs.

\section{Main results}

3 patients in the angioplasty group had revascularisation; 22 patients in the medication alone group had angioplasty. Intention to treat analyses showed that the groups did not differ at 12 months for diastolic or systo- lic BP $(160 / 93 \mathrm{~mm} \mathrm{Hg}$ for the angioplasty group $v$ $163 / 96 \mathrm{~mm} \mathrm{Hg}$ for the medication alone group, $\mathrm{p}=0.5$ ) or for the number of drug doses $(2.5 v 3.1, \mathrm{p}=0.10)$. The groups also did not differ for serum creatinine concentrations (median $1.2 \mathrm{mg} / \mathrm{dl}$ for both groups) or creatinine clearance rates $(70 v 62 \mathrm{ml} / \mathrm{min}, \mathrm{p}=0.1)$.

\section{Conclusion}

For patients who have atherosclerotic renal artery stenosis with normal or mildly impaired renal function, primary angioplasty was not more effective than antihypertensive drugs alone for reducing blood pressure or limiting disease progression.

*See glossary.

$\dagger$ Information provided by author.

\section{COMMENTARY}

This useful study by van Jaarsveld $e t a l$ addresses a common and realistic clinical problem. It expands and complements previous small studies but will not be welcomed by those who already believe in surgical intervention for renal artery stenosis. The major weakness is that, because of the relatively high recurrence rates in stenosis caused by atheroma, many interventional radiologists and clinicians have already moved on to regular stenting after successful angioplasty.

The study clearly indicates that initial angioplasty did not improve either BP control or renal function at 12 months. The study provides no information on patients with renal artery stenosis and flash pulmonary edema nor on patients with steadily worsening renal function or BP control Approximately $50 \%$ of patients assigned to drug treatment subsequently had "rescue" angioplasty, and the study does not permit us to determine whether they had better outcomes. An accompanying editorial points out these limitations and suggests that further study is required for patients with more severe renal impairment and tight renal artery stenosis either in a single kidney or bilaterally. ${ }^{1}$

C P Swainson, MB Royal Infirmary of Edinburgh Edinburgh, UK

1 Ritz E, Mann JF. Renal angioplasty for lowering blood pressure. $N$ Engl J Med 2000;342:1042-3.
Source of funding: Dutch Health Insurance Executive Board.

For correspondence: Dr B C van Jaarsveld, Dianet Dialysi. Centres, Brennerbaan 130,3524 BN Utrecht the Netherlands. Fax $+31302817094$ 